\title{
BRCA 1 and BRCA 2 Gene Sequencing in Indonesian Urban Women: Further Study of Noncommunicable Disease Research 2016
}

\author{
Kindi Adam ${ }^{1 *}$, Holy Arif Wibowo ${ }^{1}$, Rosa Adelina ${ }^{1}$, Natalie Laurencia ${ }^{1}$, Frans Dany ${ }^{1}$, \\ Setia Pramana ${ }^{2}$, Annisa Muthiah ${ }^{1}$, Rita Marleta ${ }^{1}$, Sarwo Handayani ${ }^{1}$ \\ ${ }^{1}$ Center for Research and Development of Biomedical and Basic Health Technology, National Institute for Health \\ Research and Development, Jakarta, Indonesia \\ ${ }^{2}$ Sekolah Tinggi Ilmu Statistik (STIS), Indonesia, Indonesia \\ *Corresponding author. Email: kindiad@yahoo.co.id
}

\begin{abstract}
Introduction: BRCA1 and BRCA2 are genes associated with the risk of hereditary breast cancer, one of the most cancer found in Indonesian women. The study of the genetic pattern of BRCA1 and BRCA2 genes in the Indonesian population is still very limited. Therefore, the research purpose was to identify the mutation pattern on BRCA1 and BRCA2 genes in the population of Indonesian women a part of NCD study in 2016. Method: Total of 442 buffy coat specimens met the inclusion criteria were sequenced for BRCA1 and BRCA2 whole genes using the NGS method with the Miseq Illumina platform. The data were then analyzed using appropriate software. The ethical approval was obtained from NIHRD. Result: From all specimen, 15 mutations related to the progressive benign risk and 1 VUS were discovered on the BRCA 1 gene. Meanwhile, 27 mutations known as benign, 2 VUS, and 1 low pathogenic probability were found on the BRCA2 gene. Conclusion: In the Indonesian population, there were mutation patterns on both BRCA1 and BRCA2 genes with benign and low pathogenic probability. These results can be the first step to identify the unique mutations within both genes in the Indonesian population. It also can be an initial step to design molecular diagnostic techniques, specifically for the entire Indonesian Population.
\end{abstract}

Keywords: sequencing, BRCA 1, BRCA 2, urban women, NCD, Indonesia

\section{INTRODUCTION}

Breast cancer $(\mathrm{BC})$ is the most common cancer in adult women worldwide, with over than two million new cases were detected in 2018 [1]. This is also one of the most common types of cancer found in Indonesian women [2].

It is known that BRCA 1 and BRCA2 gene are associate with the risk of hereditary breast cancer [3]. Globally, the identification of 2000 mutation positions related to the pathogenicity of both genes from a variety of ethnicities around the world has been done [4]. In the molecular level, mutation in BRCA 1 an BRCA 2 are hereditary and high penetrant to breast cancer, despite of low-level proportion. Mutation on both genes are increasing the opportunity of the $\mathrm{BC}$ risk for adult women four times higher [5].

However, study of the genetic pattern of BRCA1 and BRCA2 genes in the Indonesian population is still very limited. This research purpose was to identify the mutation pattern on BRCA1 and BRCA2 genes linked to breast cancer in the population of Indonesian women from specimen collected from nationwide Non-Communicable Disease (NCD) Research in 2016.

\section{METHOD}

Samples: Four-hundred and forty-two buffy coat specimen were selected based on the simple random sampling of the total specimen collected from Survey of NCD Research in 2016. However, 3 samples were excluded from analysis due to poor quality. The inclusion criteria were Indonesian urban women aged 25-64 years old, with the positive result of breast self-examination (SADANIS) test, followed by a cervical or ovarian cancer familial history. DNA Isolation: The DNA isolation was done using DNA Qiagen robotic kit following the manufacturer instruction. Sequencing: Sequence analysis of BRCA1 and BRCA2 genes were performed using the Next Generation Sequencing method with the Miseq @Illumina platform. Data analysis: The sequence data analysis pipeline was done using FastQC, Trimmomatic, Burrows-wheeler aligner (BWA) and continued with sequence analysis by Integrative Genome Viewer (IGV) and Genome Analysis Toolkit (GATK). The lists of mutation along with the positions were compared with the NCBI Database on https://www.ncbi.nlm.nih.gov/clinvar/?term=BRCA to identify their possible clinical significance. Ethical 
Clearance: The ethics of this research was obtained from the National Institute of Health Research and Development (NIHRD) Ethical Committee LB.02.01/2/KE.229/2018 on July 10th, 2018.

\section{RESULTS AND DISCUSSION}

From all sample examined, 17 mutation positions were detected in BRCA1 gene and a total of 30 mutation positions were spotted in BRCA2. From these, 15 known mutations were located and classified as progressive benign risk and 1 as Variance Undetermined Sequence (VUS) in the BRCA1. As for BRCA2, there were 27 mutation positions identified as benign, 2 Variance Undetermined Sequence (VUS), and 1 with low-pathogenic probability.

Table 1. List of top five mutation positions on BRCA1 gene in buffy coat specimen taken from adult women respondent of NCD research 2016.

\begin{tabular}{ccccccc} 
No & Gene & Position & Mutation & \multicolumn{2}{c}{ Number of samples } & $\begin{array}{c}\text { Proportion } \\
(\mathrm{N}=439)\end{array}$ \\
\cline { 3 - 5 } & & & Ref & Alt & & \\
\hline 1 & BRCA1 & 49155 & G & A & 178 & $40.55 \%$ \\
\hline 2 & BRCA1 & 59789 & AAAG & A & 140 & $31.89 \%$ \\
\hline 3 & BRCA1 & 59764 & AAAAAAAAAAAAG & A & 118 & $26.88 \%$ \\
\hline 4 & BRCA1 & 53052 & TA & T & 112 & $25.51 \%$ \\
\hline 5 & BRCA1 & 48124 & T & C & 81 & $18.45 \%$ \\
\hline
\end{tabular}

Table 2. List of top five mutation positions on BRCA2 gene in buffy coat specimen taken from adult women respondent of NCD research 2016.

\begin{tabular}{ccccccc}
\hline No & Gene & Position & \multicolumn{2}{c}{ Mutation } & Number of samples & $\begin{array}{c}\text { Proportion } \\
\text { nnnnnn}\end{array}$ \\
\cline { 4 - 5 } & & & Ref & Alt & & \\
\hline 1 & BRCA2 & 25389 & G & C & 357 & $81.32 \%$ \\
\hline 2 & BRCA2 & 23439 & A & G & 208 & $47.38 \%$ \\
\hline 3 & BRCA2 & 39771 & T & C & 206 & $46.92 \%$ \\
\hline 4 & BRCA2 & 31228 & T & C & 192 & $43.74 \%$ \\
\hline 5 & BRCA2 & 47030 & T & C & 75 & $17.08 \%$ \\
\hline
\end{tabular}

Table 3. Possible clinical significance of mutation from BRCA1 gene of buffy coat specimen taken from adult women respondent of NCD research 2016.

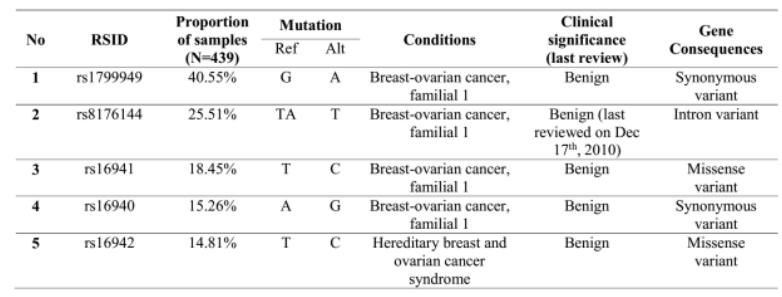

Table 4. Clinical significance of mutation from BRCA 2 gene of buffy coat specimen taken from adult women respondent of NCD research 2016.

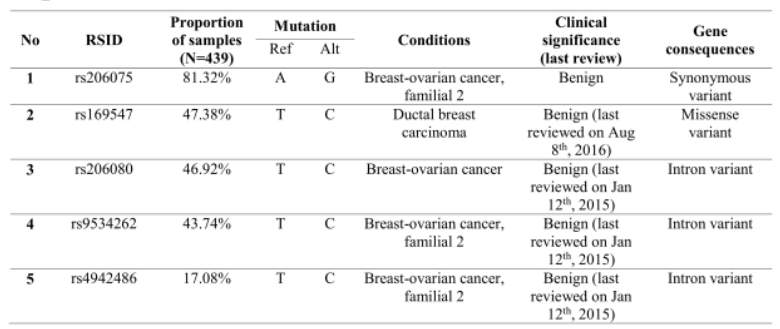

Breast cancer is one type of cancers that is multifactorial and is still not known for its definite causes. Some of the genes allegedly associated with breast cancer are BRCA1 and BRCA2. Mutations in both genes increase the likelihood of the incidence of breast cancer in women up to more than five-fold.5

There were total of 2489 BRCA1/2 mutations detected in this study. The 604 points have been confirmed with a cutoff variant quality value of 400 . These mutations were believed to have strong relationship with the risk of developing breast cancer. Mutations in both genes are still recognized as individual mutation and there is yet no research reporting that the mutation may lead to breast cancer in a particular genetic group or certain ethnic groups.

The results of this research showed differences with the previous one conducted by Streuewing6 asserting that Ashkenazi Jewish women had 10 times greater risk of breast cancer than general female population in the United States.6,7 Specific mutations in Jewish women related to breast cancer were in the BRCA1 gene (187delAG and 5385insC) and BRCA2 (6174delT).8,9 Another research report found that the Afro-American women have a lower risk of breast cancer as compared to American white women (mutation rate at $27.9 \%$ versus $46.2 \%$ ).10 Further research7 concluded that non-Jewish white tribes had a gene mutation rate of BRCA1 at 2.2\%, Hispanic (3.5\%), Afro-Americans (1.3\%) and Asian Americans $(0.5 \%)$ in women younger than 65 years who had breast cancer.

The risk classification of breast cancer based on the mutation location and its type is used in the ClinVar database [11]. The severity degree starts from the Variant Uncertain significant (VUS) category which means that the abberance significance or its risk level is not known, then mutations with low-risk or likely benign and benign [12]. Followed by high-risk category up to pathogenic level. High-risk category indicates that the variant mutation location is at bigger risk of pathogenicity. Nevertheless, at this stage, high-risk pathogenicity category is still under constant scrutiny evaluation and review. The highest level of risk is confirmed as a high pathogenicity of breast cancer [13]. 
Although the variants of BRCA1 and BRCA2 have been identified, the existence of these cannot be straightforwardly interpreted as having phenotypic manifestation, because the current reference accounts mostly for human sequencing results from Europeans or Caucasians participating in the Human Genome Project. Based on the reference obtained from the NCBI database https://www.ncbi.nlm.nih.gov/clinvar/?term=BRCA, clinical significance for several mutations were detected in this investigation.

The mutation sites of BRCA1 spotted in this research mostly were benign. Compared to the clinical variant database that has been reviewed and listed in the Web, we demonstrated 15 BRCA1 mutations associated with benign progressivity in developing breast cancer (Table 3). On the other hand, there was a mutation still expressed as a variant with unclear signification (Variance Uncertain Significant, VUS). Furthermore, there was another variant inconclusive for its level of pathogenicity due to contradicting results of existing studies one another.

In the BRCA1 gene, there were 178 (40.55\%) subjects carrying mutation in the position 43093449 of the $\mathrm{G}>\mathrm{A}$ and it's considered as benign clinical significance to developing breast cancer. This mutation is recognized as rs1799949 at the genome position of 43093449 . This position was known to be found in female patients with early onset of diagnostic breast cancer $[14,15]$ and also in Iranian male breast cancer patients with HER-2 and CK5/6 negatives [16]. Although this genetic abberance is regarded as benign, its appearance in the respondent reached $40.55 \%$ of the total specimen.

The location of another mutation in this research, namely rs8176144 is Del TA > A, was identified in 112 specimens $(25.51 \%)$. Based on previous studies, its clinical significance was also deemed benign. Another collaborative work spotted this mutation in $0.15 \%$ of the population in Africa and $0367 \%$ in the East Asian population [17].

In addition to the two spots of the mutation, there were 2 other mutation positions denoting deletion, each at the site 59789 del AAAG as many as 140 respondents (31.89\%) and one at 59764 del AAAAAAAAAAAAG in up to 118 subjects $(26.88 \%)$. The two mutation spots are not yet recorded in the list of clinical mutations in the ClinVar Database. These need to undergo thorough analysis in more advanced research employing Sanger sequencing method on the selected samples with detected deletion since the variants detected in more than $25 \%$ of the total samples.

In the BRCA2 gene, as presented in Table 4, there were 27 mutation sites with low-level breast cancer progressivity (benign). But in the BRCA2, it also showed that there was a mutation with considerable pathogenicity, although it is still at low severity (potentially pathogenic). On the other hand, the VUS mutation was also identified in two other locations.

As for BRCA2 gene in this work, there was one dominant mutation G25389C that appeared in most of the specimen $(82.32 \%)$ examined. This mutation enlisted in dbSNP and ClinVar is known as rs206076. This mutation was considered as benign with synonymous type of mutation.13,16 This $\mathrm{G}>\mathrm{C}$ mutation are in line with the most mutations found for this position compared to the minor result $\mathrm{G}>\mathrm{T}(0.005 \%)$ [18]

The other mutation unveiled from this study on BRCA2 were rs206075 with $\mathrm{A}>\mathrm{G}$ substitution (47.38\%), rs 169547 $\mathrm{T}>\mathrm{C}(46.9 \%)$ and $\mathrm{rs} 206080 \mathrm{~T}>\mathrm{C}(43.7 \%)$. These three mutations were considered benign with genetic consequences as synonymous, missense and intron variant respectively.

As for the rs206075 mutation (47.38\%), the $A>G$ mutation frequency was 0.99 for all population except American, Ashkenazi Jews and East Asian with 100\% frequency. This mutation from dbSNP were taken from breast cancer patient. This result was slightly different because this study targeted community-based respondents who were not necessarily a breast cancer patient even if they had a positive clinical breast examination result (CBE) and/or family ties to breast cancer patients. Almost half of the total sample examined had this mutation and other have ancestral nucleotides as the reference. This result along with CBE result needs to be considered in further study.

Nevertheless, the rs 169547 variance with $\mathrm{T}>\mathrm{C}$ mutation had a benign clinical significance for breast cancer but also considered as VUS for ductal breast carcinoma. This type of mutation also accounted for more than $90 \%$ specimen examined globally for genetic sequencing. The similar result was expected for rs206080 variance as well.

Based on the dbSNP database for BRCA2, two mutations from this study were classified as conflicting clinical significance. The rs80358589 spot with $A>G$ mutation was located on 4 specimens. This contradictory finding was based on various examination from other study showing that this mutation was considered as VUS for breast cancer yet also related to other cancer such as esophageal carcinoma and Fanconi anemia with likely-benign clinical significance [19-22]. On the other hand, the rs80358393 position with $A>G$ mutation was identified in one specimen. This mutation site is considered rare and found only in $1 \%$ of East Asian population. This mutation changes the amino acid translation from threonin to alanin. This mutation are categorized both as VUS and benign because of the missense translation [23,24]. To best of our knowledge, this is the first study to perform BRCA1/2 genetic sequencing using NGS method for communitybased studies in Indonesia. The result of this study reiterates the importance of NGS approach for further study to 
identify the causal mutation of breast cancer using hospitalbased samples taken from breast cancer-positive patients.

\section{CONCLUSION}

In the Indonesian population, there were mutation patterns on both BRCA1 and BRCA2 genes with benign and low pathogenic probability. The BRCA1 mutation rs1799949 was detected in $40.55 \%$ samples with clinical significance as benign. Whereas for the BRCA2 mutation on rs206076, rs206075, rs169547, rs206080 was found in $81.32 \%$, $47.38 \%, 46.92 \%$ and $43.74 \%$ of the samples respectively and also classified as benign. These results can be the first step to identify the unique mutations within both genes on a large-scale of Indonesian community. Moreover, this information also provides a basis for designing molecular screening tools, specifically for the entire Indonesian population, in attempt to support independent health technology national policy.

\section{ACKNOWLEDGMENT}

The authors would like to thank to the Head of NIRD Indonesia for the support for this Study. This work was supported by the DIPA 2018 Grant from The Center for Research and Development of Biomedical and Basic Health Technology, MOH Indonesia.

\section{REFERENCES}

[1] Ferlay, J., et al. Estimating the global cancer incidence and mortality in 2018: GLOBOCAN sources and methods. International journal of cancer, 2019, 144.8: 1941-1953.

[2] Stewart, Bernard W, Wild CP. International Agency for Research on cancer. World Health Organizarion. World cancer report 2014. World cancer Report 2014. 2014 [Internet]. 2014;(1):630. Available from: http://www.iarc.fr/

[3] Sun Y, Zhao Z, Yang Z, Xu F, Lu H, Zhu Z, et al. Risk Factors and Preventions of Breast Cancer. 2017;13.

[4] Armstrong, N., Ryder, S., Forbes, C., Ross, J., \& Quek, R. G. (2019). A systematic review of the international prevalence of BRCA mutation in breast cancer. Clinical epidemiology, 11, 543.

[5] Antoniou A, Pharoah PDP, Narod S, Risch HA, Eyfjord JE, Hopper JL, et al. Average Risks of Breast and Ovarian Cancer Associated with BRCA1 or BRCA2 Mutations Detected in Case Series Unselected for Family History: A Combined Analysis of 22 Studies. Am J Hum Genet [Internet]. 2003;72(5):1117-30. Available from:

http://linkinghub.elsevier.com/retrieve/pii/S0002 929707606405

[6] Struewing, J. P., Hartge, P., Wacholder, S., Baker, S. M., Berlin, M., McAdams, M., ... \& Tucker, M. A. (1997). The risk of cancer associated with specific mutations of BRCA1 and BRCA2 among Ashkenazi Jews. New England journal of medicine, 336(20), 1401-1408.

[7] EM J, Miron A, Gong G, al et. Prevalence of pathogenic brcal mutation carriers in 5 us racial/ethnic groups. Jama [Internet]. 2007;298(24):2869-76. Available from:
http://dx.doi.org/10.1001/jama.298.24.2869

[8] Ngai Na Chloe Co, Lai-On Chu, Joseph K. F. Chow, Joseph W. O. Tam and EKON. HPV Prevalence and Detection of Rare HPV Genotypes in Hong Kong Women from Southern China with Cytological Abnormalities. ISRN Virol [Internet]. 2013;2013(November):1-5. Available http://dx.doi.org/10.5402/2013/312706

[9] King MC, Marks JH, Mandell JB. Breast and Ovarian Cancer Risks Due to Inherited Mutations in BRCA1 and BRCA2. Science (80- ). 2003;302(5645):643-6.

[10] Nanda R, Schumm LP, Cummings S, Fackenthal JD, Sveen L, Ademuyiwa F, et al. Genetic Testing in an Ethnically Diverse Cohort of High-Risk Women. Jama [Internet]. 2005;294(15):1925. Available from: http://jama.jamanetwork.com/article.aspx?doi=1 $\underline{0.1001 / \text { jama.294.15.1925 }}$

[11] . https://www.ncbi.nlm.nih.gov/clinvar/

[12] Tram, E., Savas, S., \& Ozcelik, H. (2013) Missense variants of uncertain significance (VUS) altering the phosphorylation patterns of BRCA1 and BRCA2. PloS one, 8(5), e62468.

[13] Richards, S., Aziz, N., Bale, S., Bick, D., Das, S., Gastier-Foster, J., ... \& Voelkerding, K. (2015) Standards and guidelines for the interpretation of sequence variants: a joint consensus recommendation of the American College of Medical Genetics and Genomics and the Association for Molecular Pathology. Genetics in medicine, 17(5), 405.

[14] Hondow, H. L., Fox, S. B., Mitchell, G., Scott, R. J., Beshay, V., Wong, S. Q., \& Dobrovic, A. (2011). A high-throughput protocol for mutation scanning of the BRCA 1 and BRCA2 genes. BMC cancer, 11(1), 265.

[15] Ricks-Santi, L., McDonald, J. T., Gold, B., Dean, M., Thompson, N., Abbas, M., ... \& Dunston, G. (2017). Next generation sequencing reveals high prevalence of BRCA1 and BRCA2 variants of unknown significance in early-onset breast cancer in African American women. Ethnicity \& disease, 27(2), 169.

[16] Zahra, A. Z., Kadkhoda, S., Behjati, F., Moghaddam, F. A., Badiei, A., Sirati, F., ... \& Keyhani, E. (2016). Mutation screening of BRCA genes in 10 Iranian males with breast cancer. International journal of molecular and cellular medicine, 5(2), 114.

[17] https://www.ncbi.nlm.nih.gov/snp/

[18] https://www.ncbi.nlm.nih.gov/snp/rs206076\#freq uency tab

[19] Amendola, L. M., Dorschner, M. O., Robertson, P. D., Salama, J. S., Hart, R., Shirts, B. H., .. \& \& Bennett, J. T. (2015). Actionable exomic incidental findings in 6503 participants: challenges of variant classification. Genome research, 25(3), 305-315.

[20] Zhong, L., Zhu2\&, Z. Z., Shen, Y., Sun, G., Zhao, X., Zhang, S., ... \& Zhu, G. (1771). Frequent germline mutation in the BRCA2 gene in esophageal squamous cell carcinoma patients from a low-risk Chinese population. Asian Pacific Journal of Cancer Prevention, 12.

[21] Toh, G. T., Kang, P., Lee, S. S., Lee, D. S. C., Lee, S. Y., Selamat, S., ... \& Teo, S. H. (2008). BRCA1 
and BRCA2 germline mutations in Malaysian women with early-onset breast cancer without a family history. PloS one, 3(4), e2024.

[22] Purnomosari, D., Pals, G., Wahyono, A., Aryandono, T., Manuaba, T. W., Haryono, S. J., \& Van Diest, P. J. (2007). BRCA1 and BRCA2 germline mutation analysis in the Indonesian population. Breast cancer research and treatment, 106(2), 297-304.

[23] Nykamp, K., Anderson, M., Powers, M., Garcia, J., Herrera, B., Ho, Y. Y., ... \& Group, T. I. C. G.
(2017). Sherloc: a comprehensive refinement of the ACMG-AMP variant classification criteria. Genetics in Medicine, 19(10), 1105.

[24] Borg, Å., Haile, R. W., Malone, K. E., Capanu, M., Diep, A., Törngren, T., ... \& Mellemkjaer, L. (2010). Characterization of BRCA1 and BRCA2 deleterious mutations and variants of unknown clinical significance in unilateral and bilateral breast cancer: the WECARE study. Human mutation, 31(3), E1200-E1240. 